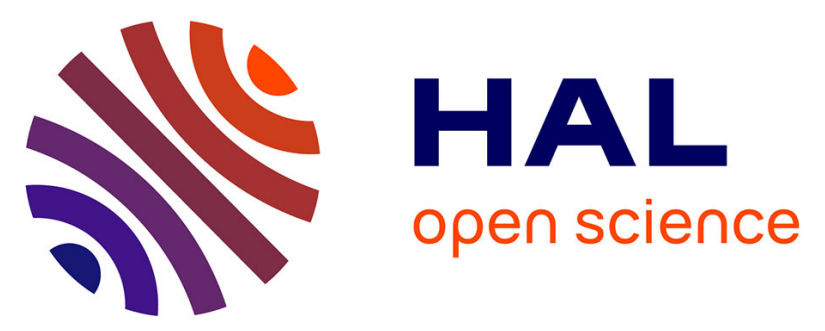

\title{
Acoustic Vibrations of Core-Shell Nanospheres: Probing the Mechanical Contact at the Metal-Dielectric Interface
}

\author{
Benoit Dacosta Fernandes, Noelia Vilar-Vidal, Hatim Baida, Pascal Massé, \\ Jean Oberlé, Serge Ravaine, Mona Tréguer-Delapierre, Lucien Saviot, Pierre \\ Langot, Julien Burgin
}

\section{To cite this version:}

Benoit Dacosta Fernandes, Noelia Vilar-Vidal, Hatim Baida, Pascal Massé, Jean Oberlé, et al.. Acoustic Vibrations of Core-Shell Nanospheres: Probing the Mechanical Contact at the Metal-Dielectric Interface. Journal of Physical Chemistry C, 2018, 122 (16), pp.9127-9133. 10.1021/acs.jpcc.7b12559 . hal-01811071

\section{HAL Id: hal-01811071 \\ https://hal.science/hal-01811071}

Submitted on 8 Jun 2018

HAL is a multi-disciplinary open access archive for the deposit and dissemination of scientific research documents, whether they are published or not. The documents may come from teaching and research institutions in France or abroad, or from public or private research centers.
L'archive ouverte pluridisciplinaire HAL, est destinée au dépôt et à la diffusion de documents scientifiques de niveau recherche, publiés ou non, émanant des établissements d'enseignement et de recherche français ou étrangers, des laboratoires publics ou privés. 


\title{
Acoustic Vibrations of Core-Shell Nanospheres: Probing the Mechanical Contact at the Metal-Dielectric Interface
}

\author{
Benoit Dacosta Fernandes, ${ }^{\dagger}$ Noelia Vilar-Vidal, ${ }^{\ddagger}$ Hatim Baida, $^{\dagger}$ Pascal Massé, ${ }^{\S}$ Jean Oberlé, $^{\dagger}$ \\ Serge Ravaine, ${ }^{\ddagger \odot}$ Mona Treguer-Delapierre, ${ }^{\S}$ Lucien Saviot, ${ }^{\| \odot}$ Pierre Langot, ${ }^{\dagger}$ and Julien Burgin ${ }^{*}{ }^{\dagger} \odot$ \\ †Université de Bordeaux, CNRS, LOMA, UMR 5798, F 33405 Talence, France \\ ‡Université de Bordeaux, CNRS, CRPP, UMR 5031, F 33600 Pessac, France \\ ${ }^{\S}$ Université de Bordeaux, CNRS, ICMCB, UMR 5026, F 33600 Pessac, France \\ "Laboratoire Interdisciplinaire Carnot de Bourgogne, UMR 6303 CNRS Université de Bourgogne Franche Comté, 9 Av. A. Savary, \\ BP 47870, F 21078 Dijon Cedex, France
}

\begin{abstract}
The acoustic vibrations of metal-dielectric core With contact -shell nanoparticles ( $\mathrm{Ag} @ \mathrm{SiO}_{2}$ and $\left.\mathrm{Au} @ \mathrm{SiO}_{2}\right)$ a $\mathrm{r}$ e investigated using ultrafast pump-probe spectroscopy. The experimental results are in good agreement with calculations of the period of the breathing vibration of the core-shell particles. The transition from one overtone of the breathing mode to the next one as the size of the silica shell increases is observed for slightly thinner $\mathrm{SiO}_{2}$ shells than previously reported. Our results confirm that this optical technique
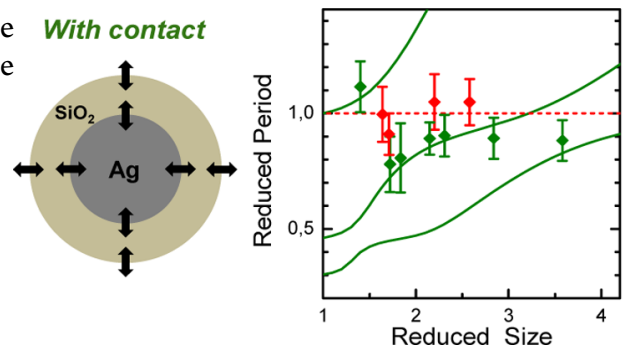

Without contact permits the determination of the quality of the mechanical contact at the metal dielectric interface. This is of major importance for many applications, in particular because it modifies significantly the thermal conductivity at the nanoscale. Moreover, we observed that for two different synthesis routes, poor contact core-shell samples could sneak in among good sample series based on the same metallic core particles. This confirms the suitability of this all optical and noninvasive technique to obtain information hardly reachable by other means such as electron microscopy or absorption spectroscopy.
\end{abstract}

\section{INTRODUCTION}

The study of the vibrational properties of nanoparticles is a fundamental subject that has been deeply investigated in the last decade. It presents a technological importance for the development of nanosystems with controlled nanomechanical and nanothermal properties. In this framework, the inves tigation of the vibrations of metallic particles and hybrid metallic-dielectric particles is of particular interest because they can be the basic building blocks of more complex nanosystems. $^{1-3}$ The investigations of the vibrations of nanosystems can yield important information concerning the object, such as its shape, ${ }^{4-9}$ size, ${ }^{5-13}$ composition, ${ }^{5,14-17}$ crystallinity, $^{18,19}$ and environment. ${ }^{5,11,20-24}$ For a liquid environment, they can serve as a local viscosity probe, ${ }^{24}$ while for a solid environment, they can act as a mechanical probe of acoustic impedances. ${ }^{22}$ The potential interest in hybrid nanosystems comes from the mix of their physical properties that can yield the emergence of new functionalities. Moreover, in terms of mechanics and acoustics, the knowledge of the interface between constituents is of major importance because it affects the properties of the whole hybrid system.

Among the methods used to investigate the vibrations, optical methods are very efficient at the nanoscale: time resolved optical spectroscopy enables the monitoring of totally symmetric low frequency acoustic modes ${ }^{5-11,17,25}$ and a few other modes are observed with Raman and Brillouin scattering. ${ }^{7,26-28}$ In the case of metallic nanosystems, optical experiments take advantage of the surface plasmon resonance (SPR) properties. ${ }^{6,9,11,29}$ The great sensitivity of the SPR observed in steady state experiments (UV-visible absorption, dark field microscopy, etc.) yields very precise information on the nano object's shape, composition, and dielectric environ ment ${ }^{4,30,31}$ but gives poor information on its internal structure. On the other hand, time resolved experiments probe the modulations of the SPR by the acoustic vibrations that are optically launched..$^{5}$ These experiments enable the measure ment of the frequency and damping of vibrational modes. Their comparison with mechanical numerical modeling allows the determination of the mechanical properties and crystallin ity. $^{5-8,10,11,18-21}$ Crystallinity can also be investigated using high resolution transmission electron microscopy (TEM), but this technique cannot assess the mechanical properties such as the quality of the mechanical contact between a metal and an amorphous dielectric that is of major importance for nano 
technological applications. A fine analysis of the time resolved measurements yields information on the quality of the mechanical contact, particularly in the case of metallicdielectric particles. $5,10,15,16$

In this study, we present results concerning the acoustic vibrations of core@shell nanoparticles $\left(\mathrm{Ag} @ \mathrm{SiO}_{2}\right.$ and $\mathrm{Au} @$ $\left.\mathrm{SiO}_{2}\right)$ synthesized through different protocols. The comparison of the measured periods with analytical modeling allows us to investigate the quality of the mechanical contact at the metaldielectric interface. We evidence that our time resolved technique can detect a lack of mechanical contact at the interface for samples that present no specific feature through classic characterization tools. We thus determine a criterion to detect the absence of mechanical contact by comparing the vibrational period measured in the core sample and core-shell samples based on the same core particles. We investigate the impact of the ligand used for core sample preparation on mechanical properties of core-shell particles, and we also investigate the hypothesis of the presence of cracks in silica.

\section{EXPERIMENTAL SECTION}

Ultrafast Spectroscopy. The time resolved pump-probe technique enables the observation of electronic and vibrational kinetics in metals. After a transient response due to electronic processes, the relative transmission changes result from electron-phonon interactions $s^{11,29,32,33}$ and later acoustic vibrations of the nanoparticles. ${ }^{5-16,18-24,29}$ This latter part is investigated here: the signal is a damped sinusoid having a period and damping time reflecting the characteristics of the particles' acoustic vibrations. The experimental pump-probe setup is based on a Ti:sapphire femtosecond laser source (Chameleon Coherent, $150 \mathrm{fs}, 680-1080 \mathrm{~nm}, 80 \mathrm{MHz}$ ). The pulse train is split into two parts. The first one, the pump pulse, is frequency doubled and used to create a nonequilibrium electron distribution in the metal. The second part, the probe pulse, is also frequency doubled and is used to monitor the induced sample transmission changes $\Delta \mathcal{T} / \mathcal{T}$ for several delay times. The probe wavelength $\left(\lambda_{\text {pr }}\right)$ is chosen in the vicinity of the SPR to maximize the sensitivity. It is always chosen on the same side of the SPR (blue side here) to get rid of potential inhomogeneous effects. $^{21,23,29}$ The pump wavelength $\left(\lambda_{\mathrm{pp}}\right)$ is chosen to be identical to the probe one to take advantage of the SPR absorption and perform a sufficiently strong excitation of the particles. We also used infrared pumping and did not notice any modification of the vibrational characteristics. The detection is performed with mechanical chopping of the pump beam at $2 \mathrm{kHz}$ and lock in differential detection of the transmitted probe beam intensity. The high stability and repetition rate of our setup with a noise level in the $10^{-7}$ range enable us to achieve high sensitivity measurements.

Chemicals. All chemicals were of analytical grade and were used without further purification. The water used in all experiments was Milli $\mathrm{Q}$ grade. Silver nitrate $\left(\mathrm{AgNO}_{3}\right.$, 99.999\%), gold chloride trihydrate $\left(\mathrm{HAuCl}_{4} \cdot 3 \mathrm{H}_{2} \mathrm{O}\right)$, sodium hydrosulfide ( $\mathrm{NaHS})$, L arginine $(\geq 98 \%)$, trisodium citrate dihydrate (NaCit) (99\%, $\left.\mathrm{Na}_{3} \mathrm{C}_{6} \mathrm{H}_{5} \mathrm{O}_{7} \cdot 2 \mathrm{H}_{2} \mathrm{O}\right)$, glycerol $\left(\mathrm{C}_{3} \mathrm{O}_{3} \mathrm{H}_{8}\right.$ ), poly (vinyl pyrrolidone) (PVP; $M_{\mathrm{w}} \approx 55000$ ), $\mathrm{O}$ [2 (3 mercaptopropionylamino)ethyl] $O^{\prime}$ methylpolyethylene glycol (PEG SH, $\left.M_{\mathrm{w}}=5000\right)$, tetraethoxysilane ( $\left.\geq 90 \%\right)$, and ammonia $\left(\mathrm{NH}_{3}, 30 \%\right)$ were purchased from Sigma Aldrich. Ethylene glycol (EG, 99\%) and ammonium hydroxide $\left(\mathrm{NH}_{4} \mathrm{OH}, 28-30\right.$ wt \%) were obtained from J.T. Baker. Absolute ethanol was obtained from VWR.
Synthesis of $\mathrm{Ag} @ \mathrm{SiO}_{2}$ Nanoparticles (Route 1). Spherical silver nanospheres (AgNPs@PVP) of radius ranging from 12.5 to $15 \mathrm{~nm}$ were synthesized following the procedure reported by Massé and co workers. ${ }^{3}$ In two different vials, 16.9 $\mathrm{mg}$ of $\mathrm{NaHS}$ was dissolved in $10 \mathrm{~mL}$ of EG and $900 \mathrm{mg}$ of PVP in $30 \mathrm{~mL}$ of EG. Both solutions were left to age for $4 \mathrm{~h}$ under magnetic stirring. After $1 \mathrm{~h} 30 \mathrm{~min}, 120 \mathrm{~mL}$ of EG was introduced in a rounded flask which was equipped with a reflux condenser, and the temperature was increased to $150{ }^{\circ} \mathrm{C}$ for $2 \mathrm{~h}$ $30 \mathrm{~min}$. An argon flux was introduced in the rounded flask with the EG for the last $30 \mathrm{~min}$ to create an inert atmosphere. After $4 \mathrm{~h}$ of aging, $70 \mu \mathrm{L}$ of the NaHS solution and $30 \mathrm{~mL}$ of the PVP solution were added. Immediately after, $10 \mathrm{~mL}$ of EG solution containing $240 \mathrm{mg}$ of silver nitrate was added into the rounded flask. After $9 \mathrm{~min}$, the reaction was quenched by introducing the rounded beaker in an ice bath and adding 50 $\mathrm{mL}$ of cold absolute ethanol. The silver nanoparticles were washed three times using a Millipore Stirred Ultrafiltration Cell with a regenerated cellulose membrane $(100 \mathrm{kDa})$. They were finally redispersed in absolute ethanol to get a final silver concentration of $1.6 \mathrm{~g} / \mathrm{L}$. In a further step, both batches of silver nanoparticles were coated with a silica layer of controlled thickness. Typically, $3.75 \mathrm{~mL}$ of the Ag nanoparticle dispersion was mixed under continuous magnetic stirring with a solution of deionized water and ammonia, at a volume ratio of 93.8/5/ 1.2 for absolute ethanol, water, and ammonia, respectively. By varying the amount of the silica precursor, core-shell particles with a wide range of silica shell thickness were synthesized. For example, for a shell thickness of $10 \mathrm{~nm}$ (AgNPs core $R_{1}=12$ $\mathrm{nm}), 20 \mu \mathrm{L}$ of tetraorthosilicate was added, and the reaction mixture was stirred for $12 \mathrm{~h}$ at $\sim 20^{\circ} \mathrm{C}$. Upon completion of the growth of the silica shell, the particles were washed with absolute ethanol and water and redispersed in water.

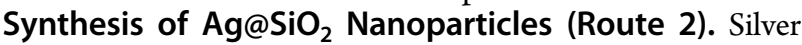
nanoparticles of radius ranging from 11.6 to $12.2 \mathrm{~nm}$ and coated with PEGSH (AgNPs@PEG SH) were prepared according to the following procedure. ${ }^{34}$ Briefly, $9 \mathrm{mg}$ of silver nitrate was added to $50 \mathrm{~mL}$ of a water/glycerol mixture (40 vol $\%$ glycerol) at $117{ }^{\circ} \mathrm{C}$. One minute later, $1 \mathrm{~mL} / 0.135 \mathrm{M}$ sodium citrate was added. Subsequently, the temperature was decreased at $97{ }^{\circ} \mathrm{C}$. After $1 \mathrm{~h}$, the solution was stored at $4{ }^{\circ} \mathrm{C}$. To prevent the oxidation of the AgNP surface and ensure the correct growth of a silica shell, the silver nanoparticles were surface functionalized by using a PEG $\mathrm{SH}$ aqueous solution $(O$ [2 (3 mercaptopropionylamino)ethyl] $O^{\prime}$ methylpolyethylene glycol $\left.M_{\mathrm{w}}=5000\right)$. A ratio of 2.7 PEG molecules per $\mathrm{nm}^{2}$ of the available surface of the silver sol was fixed. The aqueous solution of PEG SH was freshly prepared and added dropwise to the as prepared silver nanoparticles under vigorous magnetic stirring. The mixture was left to react for $1 \mathrm{~h}$ and centrifuged at $9000 \mathrm{rpm}$ for $30 \mathrm{~min}$ (twice) to eliminate remaining reactants. The AgNPs@PEG SH nanoparticles were redispersed in absolute ethanol. The deposition of silica on the metal surface was performed using the sol-gel approach described above.

Synthesis of Au@SiO ${ }_{2}$ Nanoparticles. Spherical gold nanoparticles (AuNPs) of various diameters were synthesized by a multistep procedure. AuNPs of $7 \mathrm{~nm}$ radius were first prepared by the citrate reduction method reported by Turkevich. ${ }^{35}$ AuNPs of larger radius (up to $42.5 \mathrm{~nm}$ ) were obtained by the multistep citrate reduction protocol reported by Bastùs et al. ${ }^{36} \mathrm{SiO}_{2}$ coating was carried out after surface functionalization of the AuNPs by using a PEG SH $\left(M_{\mathrm{w}}=\right.$ $5000)$ aqueous solution ( $\mathrm{C} 2$ (3 mercaptopropionyl amino) 
ethyl] $O^{\prime}$ methylpolyethylene glycol). ${ }^{37}$ The surface modifica tion allowed the replacement of the citrate molecules adsorbed onto the gold surface by the PEG SH. A ratio of 2.7 PEG molecules per $\mathrm{nm}^{2}$ of the available surface of the gold sol was fixed. The aqueous solution of PEG SH was freshly prepared and added dropwise to the as prepared AuNPs under vigorous magnetic stirring. The mixture was left to react for $1 \mathrm{~h}$ and was centrifuged at $9000 \mathrm{rpm}$ for $30 \mathrm{~min}$ (twice) to eliminate undesired reactants. The AuNPs@PEG SH nanoparticles were redispersed in absolute ethanol and further coated with silica.

Characterization. TEM images were obtained with a JEOL JEM 1400 transmission electron microscope operating at an acceleration voltage of $100 \mathrm{kV}$. Absorption spectroscopy was performed using a Shimadzu UV3600 spectrometer.

\section{RESULTS AND DISCUSSION}

The experiments were performed in colloidal solution of core Ag with radius $R_{1}$ ranging from 11.5 to $15 \mathrm{~nm}$ and core-shell $\mathrm{Ag} @ \mathrm{SiO}_{2}$ nanoparticles with a shell thickness $e=R_{2}-R_{1}$ ranging from 5 to $54 \mathrm{~nm}$ (Figure 1). The resulting range in

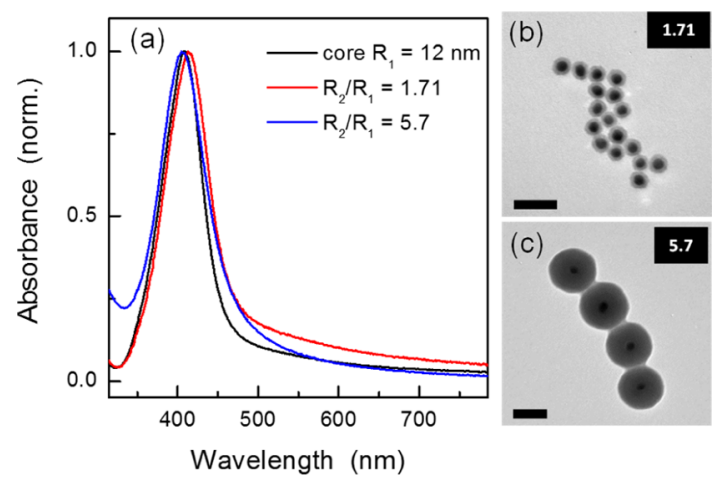

Figure 1. (a) UV-visible spectra of $\mathrm{Ag}$ core particles (radius $R_{1}=12$ $\mathrm{nm}$, black curve) and $\mathrm{Ag} @ \mathrm{SiO}_{2}$ core-shell samples (CS1 in red and CS2 blue) with total radius $R_{2}$. (b,c) TEM picture of the core-shell samples CS1 $\left(R_{1}=12 \mathrm{~nm}, R_{2}=20.5 \mathrm{~nm}\right)$ and CS2 $\left(R_{1}=11.5 \mathrm{~nm}, R_{2}\right.$ $=65.5 \mathrm{~nm})$. The numbers represent the radii ratio $R_{2} / R_{1}$. Scale bar: $100 \mathrm{~nm}$.

terms of the radius ratio $R_{2} / R_{1}$ goes from 1.4 to 5.7 . Figure 1a presents the absorption spectra of core $\left(R_{1}=12 \mathrm{~nm}\right)$ and two core-shell samples (CS1 and CS2) synthesized following route 2. The SPR of core particles (408 $\mathrm{nm}$ ) is weakly modified by silica deposition. The temporal evolution of $\Delta \mathcal{T} / \mathcal{T}$ measured in silver core particles $\left(R_{1}=12 \mathrm{~nm}\right)$ for identical pump and probe wavelength $\left(\lambda_{\mathrm{pp}}=\lambda_{\mathrm{pr}}=380 \mathrm{~nm}\right)$ is presented in Figure 2 . After a sharp rise during the first hundred femtoseconds following energy absorption by the metal, $\Delta \mathcal{T} / \mathcal{T}$ decreases exponentially, representing electron-phonon energy ex changes. $^{32,33}$ Then, $\Delta \mathcal{T} / \mathcal{T}$ presents modulations that can be associated with the vibrational modes of the nanopar ticles. $^{5,10,11,29}$ The measured periods are obtained by fitting with a function $f(t)$ which sums several modes (amplitude $A$, period $T$, damping time $\tau$, and phase $\varphi$ ) for the oscillating part of the signal and if necessary a monoexponential decay (amplitude B, decay time $\tau_{\mathrm{e}-\mathrm{ph}}$ ) for the initial electronphonon decay

$$
f(t)=\sum_{i} A_{i} \mathrm{e}^{-t / \tau_{i}} \cos \left(\frac{2 \pi}{T_{i}} t+\varphi_{i}\right)+B \mathrm{e}^{-t / \tau_{\mathrm{e}-\mathrm{ph}}}
$$

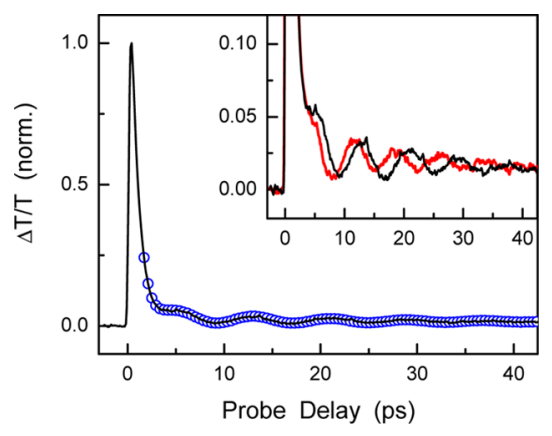

Figure 2. Normalized time resolved transient transmission of the Ag core $\left(R_{1}=12 \mathrm{~nm}\right)$ measured with $\lambda_{\mathrm{pp}}=\lambda_{\mathrm{pr}}=380 \mathrm{~nm}$. The blue dots represent the fit using eq 1. Inset: Transient transmission of the core sample $R_{1}=12 \mathrm{~nm}$ (black) and core-shell sample CS1 in red $\left(R_{2} / R_{1}\right.$ $=1.71$ ).

We find that a single vibration mode is enough for the fitting function to perfectly match the measured signal (see Figure 2). The observed vibration period is found to be $T_{\text {core }}^{\mathrm{A}}=7.90 \mathrm{ps}$; this value is in good agreement with analytical calculations (7.2 ps) for the fundamental breathing mode of a nanosphere with a $12 \mathrm{~nm}$ radius in water. ${ }^{5}$

The inset shows the measurements for a core@shell Ag@ $\mathrm{SiO}_{2}$ sample (CS1) compared to the one observed in the core. The CS1 sample presents a single mode oscillation with a period shorter than the core $\left(T_{\text {core }}^{\mathrm{A}}\right): T^{\mathrm{CS} 1}=7.20 \mathrm{ps}$. We performed experiments on several $\mathrm{Ag}$ core and $\mathrm{Ag} @ \mathrm{SiO}_{2}$ coreshell samples with different values of core radius $R_{1}$ and shell thickness $R_{2}-R_{1}$ to investigate the evolution of the observed mode for particles in a large range of the $R_{2} / R_{1}$ value: from 1.4 to 5.7. We plotted the reduced period $T^{\mathrm{CS}} / T_{\text {core }}$ as a function of $R_{2} / R_{1}$ in Figure $3 . \mathrm{W}$ e see that this ratio is close to 1 for several samples, but deviations clearly appear for several others, among them CS1 as already mentioned. To understand this behavior, we compared these results with numerical simulations. These measured periods are compared to calculations to assign them to vibration eigenmodes and extract physical information on the nanoparticles, their structure, and their environment.

We first calculated the acoustic vibration periods for nanospheres (the core particle) in several environments. Analytical calculations made by Lamb and detailed in this review $^{5}$ yield the following results. The periods of the fundamental mode vary linearly with the diameter $T=\alpha D$, where $\alpha$ (in ps $/ \mathrm{nm}$ ) is a numerical coefficient depending on the particle and environment constituents. We find $\alpha_{\mathrm{Ag}}^{\text {free }}=0.298$, $\alpha_{\mathrm{Ag}}^{\text {water }}=0.299$, and $\alpha_{\mathrm{Ag}}^{\text {silica }}=0.280$. The periods for a free particle and a particle in water are very similar because water has weak acoustic impedance compared to silver. ${ }^{5}$ The period for a particle embedded in silica is also very close to the one in water: the higher impedance has a weak effect on the period and manifests mostly on the mode damping. ${ }^{5}$ To conclude, we will use $\alpha_{\mathrm{Ag}} \approx 0.3$ for the rest of the discussion and consider that $T_{\text {core }}^{\text {water }} \approx T_{\text {core }}^{\text {silica }} \approx T_{\text {core }}^{\text {free }}$. The periods for core-shell particles were calculated as Portalès et al for many silica thicknesses. ${ }^{28}$ As expected, after normalization of the core-shell period $T^{\mathrm{CS}}$ by the breathing period of the core $T_{\text {core }}$ the reduced periods $T^{\mathrm{CS}} /$ $T_{\text {core }}$ depend only on $R_{2} / R_{1} \cdot{ }^{15,16}$ The results are presented in Figure 3. We represent the reduced period, i.e., the core-shell period normalized to the core period, as a function of the radius ratio $R_{2} / R_{1}$. The first four eigenmodes are shown $(n=0, \ldots, 3)$. 


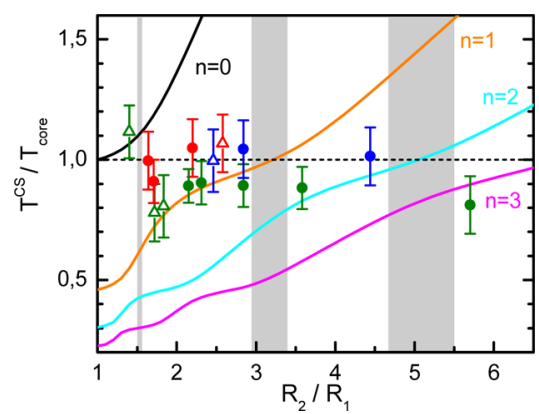

Figure 3. Reduced period ( $\left.T^{\mathrm{CS}} / T_{\text {core }}\right)$ measured in several core-shell samples as a function of the radius ratio $R_{2} / R_{1}$. The triangles and circles correspond to samples obtained with synthesis routes 1 and 2, respectively. The color code represents the contact quality estimation (green $=$ good, blue $=$ undetermined, and red $=$ poor). The reduced periods computed for the fundamental radial mode $(n=0)$ of an Ag@ $\mathrm{SiO}_{2}$ core-shell particle and for three overtones $(n=1,2$, and 3$)$ are shown by the solid lines in black, orange, cyan, and magenta, respectively. The black dashed line corresponds to the reduced period expected in the case of full core-shell decoupling (i.e., $T^{\mathrm{CS}} / T_{\text {core }}=1$ ). The gray stripes correspond to the $R_{2} / R_{1}$ window where mode switching occurs.

Calculations show that several anticrossing patterns are present as previously observed by Vallée and co workers. ${ }^{10,15,16}$ We also experimentally observe that the nanosystems vibrate along a single dominant mode because fitting the variation of the transmission requires only a single damped sine function. The reason for this behavior is to be found in the nature of the different overtones. Some of them correspond to vibrations of the metallic core. The other ones concern mainly the silica shell. However, the excitation mechanism is related to the absorption by the metallic core, and the detection is also related to variation of the transmission of the metallic core. Therefore, the pump-probe measurements are sensitive only to the vibrations whose amplitude inside the metallic core is not small. The corresponding overtone index $n$ chosen by the nano particle varies with $R_{2} / R_{1}$. We find that the nanosystem oscillates along mode $n=0$ for $R_{2} / R_{1}<1$.5. In this range, the core-shell period is bigger than the core one. Then, the system switches to mode $n=1$ near $R_{2} / R_{1}=1.5$. This value is smaller than the one measured by a previous study. ${ }^{16}$ For $1.5<R_{2} / R_{1}<$ 3 , we investigated several samples that dominantly oscillate along the mode $n=1$. The mode switch from $n=1$ to $n=2$ appears when $R_{2} / R_{1} \approx 3-3.5$. For $3.5<R_{2} / R_{1}<4.5$, we investigated two samples that present oscillations along the mode $n=2$, which is in good agreement with the calculations. The last sample, $\left(R_{2} / R_{1}=5.7\right)$, presents oscillations along the mode $n=3$, indicating that the switch from $n=2$ to $n=3$ happens for $R_{2} / R_{1} \approx 4.5-5.5$. To the best of our knowledge, this switch had not been observed before. The mode selected by the systems is difficult to predict. A fine analysis by Crut et al. considered both excitation processes (that launch preferen tially some modes) and detection processes (that enhance some modes in the detected signal) and estimated the predominant mode one should experimentally observe. ${ }^{10,15,16}$ They predicted and observed experimentally two transitions from mode $n=0$ to mode $n=2$, which is in good agreement with their model. Our observations match with this study: we also observed single mode vibrations and three mode switches when $R_{2} / R_{1}$ increases. Meanwhile, we found a slight deviation to their prediction: we observe that the mode switches to the next overtone for $R_{2} / R_{1}$ values smaller than those predicted by their model. It seems that this phenomenon is difficult to predict quantitatively and may depend either on the synthesis route used or on the mechanical quality of the contact between the core and the shell that may not be a binary value (i.e., perfect or absent).

Moreover, a closer observation of our results shows that four samples do not behave as expected (red dots, Figure 3). These core-shell samples oscillate with a period that matches the core period. This can be explained by assuming that the mechanical contact between the $\mathrm{Ag}$ core and the $\mathrm{SiO}_{2}$ shell is poor. Indeed, it has been calculated that the presence of a thin solvent layer or organic products can induce a mechanical decoupling between the core and the shell. ${ }^{15} \mathrm{We}$ can estimate the core dilatation in our experiments, considering the absorbed energy and the induced dilatation. According to a previous study ${ }^{25}$ and taking into account our experimental configuration (high laser repetition rate and weak excitation), we estimate that the temperature elevation of the core nanoparticle is between 30 and $300 \mathrm{~K}$, resulting in a particle dilatation between 0.04 and $0.4 \%$. Maximal vibration amplitudes are thus estimated to be between 0.005 and $0.05 \mathrm{~nm}$. Because the vibrations of the core-shell particles are launched by the metallic core absorbing photons, it is clear that a thin soft layer can mechanically isolate the core from its shell. This phenomenon that has been calculated by Crut et al. ${ }^{15}$ is experimentally observed here and proposed as a characterization method. Indeed, the comparison of the measured and expected periods is a unique tool to characterize the mechanical properties of nanoparticles and particularly the nature of the contact between the core and the shell. We thus adopted a color code reported on Figure 3: the green dots represent samples with a period that matches numerical simulations assuming a perfect contact $(46 \%$ of samples), the red dots represent samples with a reduced period that is close to 1 and does not match simulations assuming a perfect contact ( $20 \%$ of samples), and the blue dots represent undetermined samples: their reduced period matches both calculations assuming a perfect contact and the value 1 corresponding to a full decoupling. We notice that samples in red in Figure 3 are present for both synthetic routes. Concerning samples synthesized using route 2 (circles), it is important to remark that they present three red dots that do not come from the same Ag core sample. This indicates that the critical step relies on the silica deposition and does not depend systemati cally on the core sample: we presume that decoupling can happen to any core-shell sample even if it is based on a core that has already been used to produce a core-shell with good mechanical contact. Meanwhile, it seems that route 2 samples having PEG SH ligands on core particles are less inclined to present a good mechanical contact in between the dielectric and the metal. The PEG SH binds strongly to the silver surface via a $\mathrm{Ag}-\mathrm{S}$ bond and may block the diffusion of the silica precursor directly to the metal surface. In contrast, with PVP as surface priming (route 1), the silica coating is promoted directly on the metal surface because PVP is weakly linked to metal through both $\mathrm{C}=\mathrm{O}$ and $\mathrm{C}-\mathrm{N}$ groups. ${ }^{38,39}$ Moreover, PVP has also a high affinity for silica, which arises from hydrogen bonding and from electrostatic interactions between positively charged PVP and silica that contains some negative charges. The structure of the PVP, particularly N,N dialkyl substituted carboxylamide, is a frequent functionality among polymers for affinity for silica. The oxygen of the carbonyl is a strong hydrogen bond acceptor and 
hence interacts well with strong hydrogen bond donators such as silanol groups. Such interactions may explain the better mechanical contact on the metal-PVP surface than on the metal PEG SH one. Considering this, it seems that during the surface modification of the core particle needed to grow the silica shell, the strong thiol binding reduces the ligand exchange efficiency and induces the presence of thin soft layers between the core and the shell that are responsible for mechanical decoupling. Therefore, measurements of acoustic vibrations of core-shell particles are a powerful tool to detect ineffective mechanical contact that may be present for samples that do not present any notable characteristics using standard characterizations such as TEM or UV-visible spectroscopy. Our technique can also be used to detect particles presenting poor mechanical contact and may be useful for specific nanotechnological applications.

The calculation of the core-shell frequencies presented in Figure 3 assumes a perfect mechanical contact as discussed above. The presence of a thin shell layer modifies the frequencies of the metallic core in two ways: (i) the mass of the shell slows down the vibrations of the core and (ii) the additional energy required to increase or to decrease the volume of the shell as the volume of the core varies results in a shorter oscillation period. This second mechanism relies on the assumption that the elasticity of thin silica shells is the same as the one of bulk silica and that the shell covers perfectly the metallic core. For completeness, we tested this assumption by considering the opposite limiting case where the elasticity of the shell vanishes and therefore only its mass plays a role. This would be the case, for example, if in our samples the silica shell contained many cracks but were nonetheless stuck to the core. This mass loaded nanoparticle hypothesis is similar to recent works on semiconductor nanoparticles ${ }^{40}$ and nanoplatelets ${ }^{41}$ coated by ligands. We calculated the periods using the model with vanishing surface stress tensor, as presented by Huang and $\mathrm{Liu}^{42}$ and the results are presented in Figure 4. This "mass

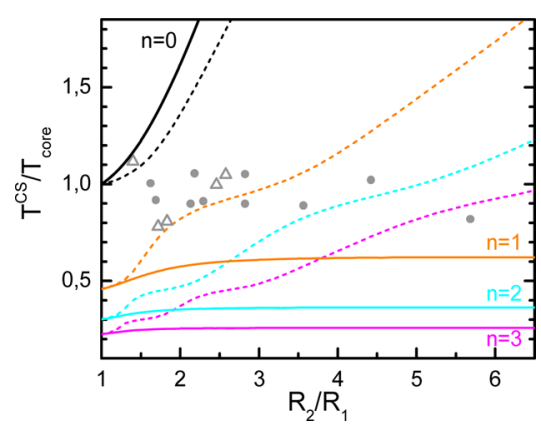

Figure 4. Reduced period ( $\left.T^{\mathrm{CS}} / \mathrm{T}_{\text {core }}\right)$ of an $\mathrm{Ag} @ \mathrm{SiO}_{2}$ core-shell particle, using the mass loaded nanoparticle hypothesis, computed for the fundamental radial mode $(n=0)$ and for three overtones $(n=1,2$, and 3) are shown by the solid lines in black, orange, cyan, and magenta, respectively. The dashed lines correspond to the computed reduced periods, assuming a perfect contact and shell elasticity as in Figure 3. Reduced period $\left(T^{\mathrm{CS}} / T_{\text {core }}\right)$ measured in core-shell samples as a function of the radius ratio $R_{2} / R_{1}$ is presented in gray dots.

loaded hypothesis" results in an increase of the period for the fundamental breathing mode $n=0$, while for the periods of the overtones $(n>0)$, it results in a decrease relatively to the previous calculations with perfect mechanical contact and the presence of shell elasticity (dashed lines, Figure 4). We also notice that as the mass of the shell increases (i.e., $R_{2} / R_{1}$ increase), the overtone periods asymptotically converge toward the periods of a sphere with a fixed surface (vanishing displacement instead of vanishing stress at the surface). Overall, we clearly see from Figure 4 that the gap between the $n=0$ and $n=1$ branches increases with the mass loaded nanoparticle hypothesis and thus does not match at all our experimental results.

We extended these investigations to $\mathrm{Au} @ \mathrm{SiO}_{2}$ nanoparticles. The core radius $R_{1}$ was between 7 and $42.5 \mathrm{~nm}$, and the shell thickness was between 7 and $24.5 \mathrm{~nm}$, yielding $R_{2} / R_{1}$ values between 1.37 and 2.5 (Supporting Information, Table S2). We measured the vibrations for core particles and core-shell particles, and we present the reduced period in Figure 5. All the

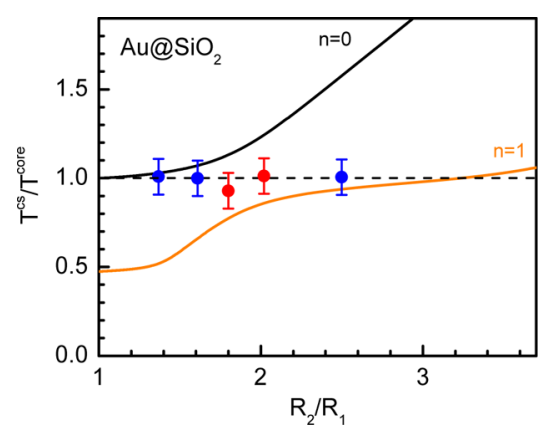

Figure 5. Reduced period $\left(T^{\mathrm{CS}} / T_{\text {core }}\right)$ measured in several $\mathrm{Au} @ \mathrm{SiO}_{2}$ core-shell samples as a function of the radius ratio $R_{2} / R_{1}$ (circles). The color code represent the contact quality estimation (green $=$ good, blue $=$ undetermined, and red $=$ bad). The reduced periods computed for the fundamental radial mode $(n=0)$ and the first harmonic $(n=1)$ of an $\mathrm{Au} @ \mathrm{SiO}_{2}$ core-shell particle are shown by the black and orange solid lines, respectively (assuming perfect shell contact and shell elasticity). The black dashed line corresponds to the reduced period expected in the case of full core-shell decoupling (i.e., $\left.T^{\mathrm{CS}} / T_{\text {core }}=1\right)$.

measured vibrations have a reduced period close to 1 . The comparison with analytical calculations of the fundamental mode $(n=0)$ and its first harmonic $(n=1)$ shows that two investigated samples present a poor mechanical contact, while three of them are undetermined. The fact that all samples present the same period leads us to believe that the full batch presents a poor mechanical contact. This reinforces our opinion concerning the role of ligands and in particular the fact that PEG SH is not perfectly effective to produce core-shell samples with a good mechanical contact. Further investigations including more relevant statistics are needed to investigate precisely the role of polymer ligands (chemical binding groups, length, and molecular weight) and determine if the quality of mechanical contact principally depends on the core particle nature or its surface functionalization.

\section{CONCLUSIONS}

We investigated the acoustic vibration of hybrid metal-silica nanoparticles and found that the particle vibrations, associated with analytical simulations, are a unique tool to characterize the mechanical contact between the metal and silica at the nanoscale. Indeed, periods in core-shell particles present deviations from the core period when the mechanical contact is good while the absence of contact can be detected by the absence of modification of the measured period. Our investigation illustrates the fact that the mechanical contact is not necessarily identical for core-shell samples based on the 
same core particle, for both synthesis routes. This emphasizes the fact that time resolved spectroscopy is a powerful tool to investigate and characterize the nature of mechanical contacts that cannot be determined with standard techniques such as UV-visible spectroscopy or TEM microscopy. The comparison of experimental data and numerical computations also confirmed that our measurements cannot be interpreted by the presence of cracks in silica that would quench its elasticity. This investigation also shows that when the mechanical contact is good, the selected mode among the possible harmonics can be mode $n+1$, while mode $n$ is expected from the theory. This may be related to a nonperfect mechanical contact and requires further investigations to be fully interpreted.

\section{AUTHOR INFORMATION}

\section{Corresponding Author}

*E mail: julien.burgin@u bordeaux.fr.

\section{ORCID}

Serge Ravaine: 0000000263438793

Lucien Saviot: 0000000212492730

Julien Burgin: 0000000186483346

\section{Author Contributions}

The manuscript was written through contributions of all authors. All authors have given approval to the final version of the manuscript.

\section{Notes}

The authors declare no competing financial interest.

\section{ACKNOWLEDGMENTS}

The authors acknowledge the Centre National de la Recheche Scientifique (CNRS) and the Conseil Régional d'Aquitaine (Project Nano Trans no. \# 20111101010) for funding. The support of the "Agence Nationale pour la Recherche" for the DIRAN project (contract \# ANR 11 NANB 0001) is gratefully acknowledged. The authors acknowledge the LOMA for the use of NSI platform.

\section{REFERENCES}

(1) van Blaaderen, A. Materials Science: Colloids Get Complex. Nature 2006, 439, 545-546.

(2) Glotzer, S. C.; Solomon, M. J. Anisotropy of Building Blocks and Their Assembly into Complex Structures. Nat. Mater. 2007, 6, 557562.

(3) Massé, P.; Mornet, S.; Duguet, E.; Tréguer Delapierre, M.; Ravaine, S.; Iazzolino, A.; Salmon, J. B.; Leng, J. Synthesis of Size Monodisperse Spherical Ag@SiO2 Nanoparticles and 3 D Assembly Assisted by Microfluidics. Langmuir 2013, 29, 1790-1795.

(4) Kelly, K. L.; Coronado, E.; Zhao, L. L.; Schatz, G. C. The Optical Properties of Metal Nanoparticles: The Influence of Size, Shape, and Dielectric Environment. J. Phys. Chem. B 2003, 107, 668-677.

(5) Crut, A.; Maioli, P.; Del Fatti, N.; Vallée, F. Acoustic Vibrations of Metal Nano Objects: Time Domain Investigations. Phys. Rep. 2015, $549,1-43$.

(6) Major, T. A.; Lo, S. S.; Yu, K.; Hartland, G. V. Time Resolved Studies of the Acoustic Vibrational Modes of Metal and Semi conductor Nano Objects. J. Phys. Chem. Lett. 2014, 5, 866-874.
(7) Burgin, J.; Langot, P.; Arbouet, A.; Margueritat, J.; Gonzalo, J.; Afonso, C. N.; Vallée, F.; Mlayah, A.; Rossell, M. D.; Van Tendeloo, G. Acoustic Vibration Modes and Electron-Lattice Coupling in Self Assembled Silver Nanocolumns. Nano Lett. 2008, 8, 1296-1302.

(8) Dacosta Fernandes, B.; Spuch Calvar, M.; Baida, H.; Tréguer Delapierre, M.; Oberlé, J.; Langot, P.; Burgin, J. Acoustic Vibrations of Au Nano Bipyramids and Their Modification under Ag Deposition: A Perspective for the Development of Nanobalances. ACS Nano 2013, 7, $7630-7639$

(9) Kirschner, M. S.; Lethiec, C. M.; Lin, X. M.; Schatz, G. C.; Chen, L. X.; Schaller, R. D. Size Dependent Coherent Phonon Plasmon Modulation and Deformation Characterization in Gold Bipyramids and Nanojavelins. ACS Photonics 2016, 3, 758-763.

(10) Crut, A.; Maioli, P.; Del Fatti, N.; Vallée, F. Time Domain Investigation of the Acoustic Vibrations of Metal Nanoparticles: Size and Encapsulation Effects. Ultrasonics 2015, 56, 98-108.

(11) Hartland, G. V. Optical Studies of Dynamics in Noble Metal Nanostructures. Chem. Rev. 2011, 111, 3858-3887.

(12) Juvé, V.; Crut, A.; Maioli, P.; Pellarin, M.; Broyer, M.; Del Fatti, N.; Vallee, F. Probing Elasticity at the Nanoscale: Terahertz Acoustic Vibration of Small Metal Nanoparticles. Nano Lett. 2010, 10, 18531858.

(13) Varnavski, O.; Ramakrishna, G.; Kim, J.; Lee, D.; Goodson, T. Optically Excited Acoustic Vibrations in Quantum Sized Monolayer Protected Gold Clusters. ACS Nano 2010, 4, 3406-3412.

(14) Stoll, T.; Maioli, P.; Crut, A.; Burgin, J.; Langot, P.; Pellarin, M.; Sánchez Iglesias, A.; Rodríguez González, B.; Liz Marzán, L. M.; Del Fatti, N.; et al. Ultrafast Acoustic Vibrations of Bimetallic Nano particles. J. Phys. Chem. C 2015, 119, 1591-1599.

(15) Crut, A.; Juvé, V.; Mongin, D.; Maioli, P.; Del Fatti, N.; Vallée, F. Vibrations of Spherical Core Shell Nanoparticles. Phys. Rev. B: Condens. Matter Mater. Phys. 2011, 83, 205430.

(16) Mongin, D.; Juvé, V.; Maioli, P.; Crut, A.; Del Fatti, N.; Vallée, F.; Sánchez Iglesias, A.; Pastoriza Santos, I.; Liz Marzán, L. M. Acoustic Vibrations of Metal-Dielectric Core-Shell Nanoparticles. Nano Lett. 2011, 11, 3016-3021.

(17) Yu, S.; Zhang, J.; Tang, Y.; Ouyang, M. Engineering Acoustic Phonons and Electron-Phonon Coupling by the Nanoscale Interface. Nano Lett. 2015, 15, 6282-6288.

(18) Hu, M.; Hillyard, P.; Hartland, G. V.; Kosel, T.; Perez Juste, J.; Mulvaney, P. Determination of the Elastic Constants of Gold Nanorods Produced by Seed Mediated Growth. Nano Lett. 2004, 4, 2493-2497.

(19) Crut, A.; Maioli, P.; Fatti, N. D.; Vallée, F. Anisotropy Effects on the Time Resolved Spectroscopy of the Acoustic Vibrations of Nanoobjects. Phys. Chem. Chem. Phys. 2009, 11, 5882-5888.

(20) Major, T. A.; Crut, A.; Gao, B.; Lo, S. S.; Fatti, N. D.; Vallée, F.; Hartland, G. V. Damping of the Acoustic Vibrations of a Suspended Gold Nanowire in Air and Water Environments. Phys. Chem. Chem. Phys. 2013, 15, 4169-4176.

(21) Yu, K.; Zijlstra, P.; Sader, J. E.; Xu, Q. H.; Orrit, M. Damping of Acoustic Vibrations of Immobilized Single Gold Nanorods in Different Environments. Nano Lett. 2013, 13, 2710-2716.

(22) Voisin, C.; Del Fatti, N.; Christofilos, D.; Vallée, F. Time Resolved Investigation of the Vibrational Dynamics of Metal Nanoparticles. Appl. Surf. Sci. 2000, 164, 131-139.

(23) Ruijgrok, P. V.; Zijlstra, P.; Tchebotareva, A. L.; Orrit, M. Damping of Acoustic Vibrations of Single Gold Nanoparticles Optically Trapped in Water. Nano Lett. 2012, 12, 1063-1069.

(24) Pelton, M.; Chakraborty, D.; Malachosky, E.; Guyot Sionnest, P.; Sader, J. E. Viscoelastic Flows in Simple Liquids Generated by Vibrating Nanostructures. Phys. Rev. Lett. 2013, 111, 244502.

(25) Hartland, G. V. Coherent Vibrational Motion in Metal Particles: Determination of the Vibrational Amplitude and Excitation Mecha nism. J. Chem. Phys. 2002, 116, 8048-8055. 
(26) Girard, A.; Gehan, H.; Crut, A.; Mermet, A.; Saviot, L.; Margueritat, J. Mechanical Coupling in Gold Nanoparticles Super molecules Revealed by Plasmon Enhanced Ultralow Frequency Raman Spectroscopy. Nano Lett. 2016, 16, 3843-3849.

(27) Still, T.; Sainidou, R.; Retsch, M.; Jonas, U.; Spahn, P.; Hellmann, G. P.; Fytas, G. The "Music" of Core-Shell Spheres and Hollow Capsules: Influence of the Architecture on the Mechanical Properties at the Nanoscale. Nano Lett. 2008, 8, 3194-3199.

(28) Portalès, H.; Saviot, L.; Duval, E.; Gaudry, M.; Cottancin, E.; Pellarin, M.; Lermé, J.; Broyer, M. Resonant Raman Scattering by Quadrupolar Vibrations of Ni Ag Core Shell Nanoparticles. Phys. Rev. B: Condens. Matter Mater. Phys. 2002, 65, 165422.

(29) Crut, A.; Maioli, P.; Fatti, N. D.; Vallée, F. Optical Absorption and Scattering Spectroscopies of Single Nano Objects. Chem. Soc. Rev. 2014, 43, 3921-3956.

(30) Mayer, K. M.; Hafner, J. H. Localized Surface Plasmon Resonance Sensors. Chem. Rev. 2011, 111, 3828-3857.

(31) Zijlstra, P.; Paulo, P. M. R; Orrit, M. Optical Detection of Single Non Absorbing Molecules Using the Surface Plasmon Resonance of a Gold Nanorod. Nat. Nanotechnol. 2012, 7, 379-382.

(32) Arbouet, A.; Voisin, C.; Christofilos, D.; Langot, P.; Fatti, N. D.; Vallée, F.; Lermé, J.; Celep, G.; Cottancin, E.; Gaudry, M.; et al. Electron Phonon Scattering in Metal Clusters. Phys. Rev. Lett. 2003, 90, 177401.

(33) Fernandes, B. D.; Le Beulze, A.; Moroté, F.; Oberlé, J.; Tréguer Delapierre, M.; Burgin, J.; Langot, P. Electron-Phonon Scattering in 2D Silver Nanotriangles. J. Phys. Chem. C 2013, 117, 22041-22045.

(34) Steinigeweg, D.; Schlücker, S. Monodispersity and Size Control in the Synthesis of 20-100 Nm Quasi Spherical Silver Nanoparticles by Citrate and Ascorbic Acid Reduction in Glycerol-water Mixtures. Chem. Commun. 2012, 48, 8682-8684.

(35) Turkevich, J.; Stevenson, P. C.; Hillier, J. A Study of the Nucleation and Growth Processes in the Synthesis of Colloidal Gold. Discuss. Faraday Soc. 1951, 11, 55-75.

(36) Bastús, N. G.; Comenge, J.; Puntes, V. Kinetically Controlled Seeded Growth Synthesis of Citrate Stabilized Gold Nanoparticles of up to $200 \mathrm{~nm}$ : Size Focusing versus Ostwald Ripening. Langmuir 2011, 27, 11098-11105.

(37) Fernández López, C.; Mateo Mateo, C.; Álvarez Puebla, R. A.; Pérez Juste, J.; Pastoriza Santos, I.; Liz Marzán, L. M. Highly Controlled Silica Coating of PEG Capped Metal Nanoparticles and Preparation of SERS Encoded Particles. Langmuir 2009, 25, 1389413899.

(38) Graf, C.; Vossen, D. L. J.; Imhof, A.; van Blaaderen, A. A General Method To Coat Colloidal Particles with Silica. Langmuir 2003, 19, 6693-6700.

(39) Ullah, M. H.; Hossain, T.; Ha, C. S. Kinetic Studies on Water Soluble Gold Nanoparticles Coordinated to Poly(Vinylpyrrolidone): Isotropic to Anisotropic Transformation and Morphology. J. Mater. Sci. 2011, 46, 6988-6997.

(40) Mork, A. J.; Lee, E. M. Y.; Dahod, N. S.; Willard, A. P.; Tisdale, W. A. Modulation of Low Frequency Acoustic Vibrations in Semiconductor Nanocrystals through Choice of Surface Ligand. J. Phys. Chem. Lett. 2016, 7, 4213-4216.

(41) Girard, A.; Saviot, L.; Pedetti, S.; Tessier, M. D.; Margueritat, J.; Gehan, H.; Mahler, B.; Dubertret, B.; Mermet, A. The Mass Load Effect on the Resonant Acoustic Frequencies of Colloidal Semi conductor Nanoplatelets. Nanoscale 2016, 8, 13251-13256.

(42) Huang, G. Y.; Liu, J. P. Effect of Surface Stress and Surface Mass on Elastic Vibrations of Nanoparticles. Acta Mech. 2013, 224, 985994. 